[6] N. Jacobson, Lie Algebras, Interscience, New York 1962.

[7]. M. I. Kargapolov, Generalized soluble groups, Algebra i Logika 2 (1963), pp. 19-28.

[8] A. G. Kuroš, Theory of Groups, vol. II, Chelsea, New York 1956.

[9] M. Lazard, Problemes d'extension concernant les $N$-groupes: inversion de la formule de Hausdorff, C. R. Acad. Sci. Paris 237 (1953), pp. 1377-1379.

[10] E. M. Levich and A. I. Tokarenko, A note on locally nilpotent torsion-free groups, Sibirsk. Mat. Ž. 11 (1970), pp. 1406-1408. Translated in Siberian Math. J. 11 (1970), pp. 1033-1034.

[11] A. I, Mal'cev, Nilpotent torsion-free groups, Izv. Akad. Nauk. SSSR Ser. Mat. 13 (1949), pp. 201-212.

[12] - Generalized nilpotent algebras and their adjoint groups, Mat. Sbornik 25 (1949), pp. $347-366$.

[13] D. J. S. Robinson, Infinite soluble and nilpotent groups, QMC Mathematics Notes, London 1969.

[14] L. A. Simonjan, Two radicals of Lie algebras, Dokl. Akad. Nauk. SSSR 157 (1964, pp. 281-283. Translated Soviet Math. Doklady 5 (1964), pp. 941-944.

[15] I. N. Stewart, An algebraic treatment of Mal'cev's theorems concerning nilpotent Lie groups and their Lie algebras, Compositio Math. 22 (1970), pp. 289-312.

[16] - Structure theorems for a class of locally finite Lie algebras, Proc. London Math. Soc. 24 (3) (1972) 79-100.

[17] R. G. Swan, Representations of polycyclic groups, Proc. Amer. Math. Soc. 18 (1967), pp. $573-574$.

MATHEMATICS INSTITUTE

UNIVERSITY OF WARWICK

Coventry

Accepté par la Rédaction le 23, 6. 1975

\title{
Properties of connected functions in terms of their levels *
}

by

K. M. Garg (Edmonton, Alberta)

Abstract. Let $f$ be a connected real-valued function defined on a connected, locally connected, Hausdorff space $X$. In this paper we investigate necessary and sufficient conditions on the levels of $f$ under which $f$ is continuous, monotone or injective, and obtain some structural properties of $f$ when it is nowhere monotone.

Among the main results, $f$ is proved to be always continuous relative to the closure of the union $S$ of connected levels of $f$. If $f^{-1}$ preserves relatively compact sets, and either $S$ is dense in $X$, or its image $f(S)$ is dense in the range of $f$, then $f$ is proved to be continuous, monotone and proper. When $f$ assumes a dense set of its values only once, it is found to be continuous and monotone, When $f$ assumes a dense set of its values only once, it is found to be continuous and monotone,
and when the singleton levels of $f$ are dense in $X, f$ is even injective. If $X$ is second countable and $f$ is nowhere monotone, it is proved that the level $f^{-1}(\alpha)$ is dense-in-itself for a residual set of values of $\alpha$ in $R$, and there further exists a residual set of points $x$ in $X$ such that $x$ is a limit point of the level $f^{-1}\{f(x)\}$. Some earlier results on the distribution of closed, connected, singleton, dense-initself and perfect levels of $f$ are also extended to the present setting.

1. Introduction. Before discussing the results of this paper, we first give some definitions that are used throughout the paper.

1.1. Definttion. The space of real numbers is denoted as usual by $R$. If $E \subset R$, a point $x \in R$ is said to be a bilateral limit point of $E$ if it is a limit point of $E$ from both the sides, and we call $E$ bilaterally closed if it contains all of its bilateral limit points. Any set $E$ is said to be singleton if it contains one and only one point, and $E$ is called countable if it is finite or countably infinite. When $E$ is a subset of a topological space $X, E$ is called a boundary set [8] if its interior is empty, $E$ is meager if it is a countable union of nowhere dense sets, and $E$ is residual if its complement $X-E$ is meager.

Let $X, Y$ be two topological spaces and $f$ be a function mapping $X$ into $Y$.

1.2. Definition. For every $y \in Y$, the set

$$
f^{-1}(y)=\{x \in X: f(x)=y\}
$$

* This paper was presented at the Conference on Monotone Mappings and Open Mappings, State University of New York, Binghamton, 1970 under the title "Monotonicity of connected functions". The research was partially supporteg by the National Research Council of Canada functions". The research
under grant no. A-4826.

2 - Fundamenta Mathematicae XCVII 
is called a level [4] of $f$ (also known as a "point inverse" or a "fiber" of $f$ ). If $\theta$ is any property of sets, let $S_{\theta}(f)$ denote the union of those levels of $f$ which have property $\theta$, and let $Y_{\theta}(f)$ be the set of elements $y$ of $Y$ for which the level $f^{-1}(y)$ has property $\theta$. These sets $S_{\theta}(f)$ and $Y_{\theta}(f)$ may be called the domain and the range of levels of $f$ with property $\theta$ respectively, and it is clear that $S_{0}(f)$ $=f^{-1}\left\{Y_{\theta}(f)\right\}$. The properties of being closed, connected, singleton, dense-initself and perfect are denoted in turn by $k, c, 1, d$ and $p$ respectively [4].

1.3. Definition. The function $f$ is said to be connected if the set $f(C)$ is connected for every connected set $C$ in $X$, and $f$ is weakly connected [7] (or "connected $(C, 0)$ " [6] if $f(C)$ is connected at least for open connected sets $C$ in $X$.

It may be remarked that every linear operator between two Hausdorff topological vector spaces $X$ and $Y$ is weakly connected, and so is in turn every seminorm on $X$ (see Hrycay [6]).

As we are interested here in connected functions, the usual hypothesis of continuity (or of onto) is not included in the following definitions:

1.4. Defintion. The function $f$ is called

(a) monotone if the set $f^{-1}(C)$ is connected for every connected set $C$ in $Y$ (see Kuratowski [9], p. 131),

(b) weakly monotone [19] if each of its levels is connected,

(c) Morrey monotone if each of its levels is compact and connected, and

(d) light [18] if every nonempty level of $f$ is totally disconnected (i.e. each of its components is singleton).

It is, in fact, a (continuous onto) Morrey monotone function that is usually called a "monotone" function (see [17], [18], [19]). To distinguish this notion from the one in (a), we refer to it as a Morrey monotone function after C. B. Morrey [12] who introduced the definition. A weakly monotone function is also known as a "monotone" [13] or a "semi-monotone" [17] function.

The properties of monotonicity and Morrey monotonicity are clearly not comparable in general, although both of them imply weak monotonicity. The usual notion of monotonicity of a function $f: R \rightarrow R$ coincides with the monotonicity in (a), but is again not comparable with Morrey monotonicity; in case $f$ is connected, its usual monotonicity is also equivalent to weak monotonicity (see Proposition 3.7). However, for a continuous function $f$ from a compact space $X$ onto a Hausdorff space $Y$ all the above three notions (a), (b), (c) of monotonicity become equivalent (see Whyburn [18], p. 138).

1.5. Definitron. If $P$ is any global property of functions, the function $f$ is said to be nowhere $P$ [5] when it does not possess $P$ on any nonempty open subspace of $X$.

Throughout the paper, unless otherwise stated, $X$ is assumed to be a locally connected space and $f$ is assumed to be a weakly connected real-valued function on $X$. For $X=R$ most of the results may be found in [2], [3] and [4].
We first consider in Section 2 the closed levels and continuity of $f$. The range $Y_{k}(f)$ of closed levels of $f$ is proved in Theorem 2.2 to be bilaterally closed (see Definitions 1.1, 1.2). In Theorem 2.5 we obtain semicontinuity, continuity, oscillation and relative continuity of $f$ in terms of its closed levels. For example, if $\alpha$ is a bilateral limit point of $Y_{k}(f)$, then $f$ is continuous at every point of the level $f^{-1}(\alpha)$. This yields a theorem of Lipiński [10] as a corollary, viz. $f$ is continuous whenever $Y_{k}(f)$ is dense in $R$. If $f$ is any connected function with a $T_{1}$ range, it is proved in Theorem 2.9 that $f^{-1}$ preserves "composed" sets, viz. sets whose components are closed. Consequently, the above function $f$ is continuous whenever it is weakly monotone and connected.

Section 3 deals with the connected levels and monotonicity of $f$. Suppose $X$ is further connected and $f: X \rightarrow R$ is also connected. In Theorem 3.3 two suitable conditions are obtained under which the set $Y_{c}(f)$ is bilaterally closed. It is proved in Theorem 3.6 that $f$ is continuous relative to the closure of the domain $S_{c}(f)$ of connected levels of $f$. According to Proposition 3.7, $f$ is monotone whenever it is weakly monotone. In case $X$ is $\sigma$-coherent (see Definition 3.1), $f$ is proved in Theorem 3.9 to be monotone when the set $Y_{c}(f)$ is dense in $R$, or the set $S_{c}(f)$ is dense in $X$. If on the other hand $X$ is Hausdorff and $f^{-1}$ preserves relatively compact sets, then under each of the two density conditions $f$ is found to be monotone, Morrey monotone and proper (Theorem 3.10).

Section 4 is devoted to the singleton levels and injectivity of $f$. Let $X$ be connected and Hausdorff. If $X$ is further separable, then the set $Y_{1}(f)$ is of the form $F-C$, where $F$ is closed and $C$ is countable (Theorem 4.4). As for the monotonicity the set $Y_{1}(f)$ turns out to be more manageable than $Y_{c}(f)$. Without any of the hypotheses of Theorems 3.9 and 3.10, $f$ is found to be monotone whenever it assumes a dense set of its values only once, and when $f$ is further nowhere constant then it also becomes injective (Theorem 4.6). If, on the other hand, $f$ is connected and the set $S_{1}(f)$ is dense in $X$, then $f$ is always monotone and injective (Theorem 4.8).

Finally, in Section 5, we study the dense-in-itself levels and nowhere monotonicity of $f$. Suppose $X$ is Hausdorff and second countable. The sets $Y_{d}(f)$ and $Y_{p}(f)$ are proved to be both of the form $G_{\delta} \cup C$, where $C$ is countable (Theorem 5.1). If the set $S_{d}(f)$ is dense in $X$, or $X$ is dense-in-itself and the set $Y_{d}(f)$ is dense in $R$, the function $f$ is found to be nowhere injective, and it becomes nowhere weakly monotone when it is further light. The set $Y_{d}(f)$ is further proved to be residual in $R$ whenever $f$ is nowhere monotone (Theorem 5.5). In case $f$ is connected and nowhere monotone, or nowhere injective, then according to Theorem 5.9 there exists a residual set of points $x$ in $X$ such that $x$ is a limit point of the level $f^{-1}\{f(x)\}$. The last two theorems assume a more general and stronger form when $X$ is locally cyclicly connected (see Definition 5.11 and Proposition 5.13).

It has been recently proved by Lipiński [11] that the above properties of the sets $Y_{\theta}(f)(\theta=k, c, 1, d, p)$ characterize these sets in the case when $X=R$. 
2. Closed levels and continuity. The results of this section are relatively simple but essential for the following sections. Let us recall our standard hypothesis that $f$ is a weakly connected real-valued function on a locally connected space $X$. If $(\alpha, \beta)$ is a finite or infinite subinterval of $R$, the inverse set $f^{-1}((\alpha, \beta))$ will be denoted simply by $f^{-1}(\alpha, \beta)$, and the same applies to the closed and semi-closed subintervals of $R$.

2.1. LEMMA. For every $\alpha \in R$ the following are equivalent:

(a) the level $f^{-1}(\alpha)$ is closed,

(b) the sets $f^{-1}(-\infty, \alpha)$ and $f^{-1}(\alpha, \infty)$ are open,

(c) the sets $f^{-1}(-\infty, \alpha]$ and $f^{-1}[\alpha, \infty)$ are closed.

Proof. Suppose (a) holds and $x$ is any point of the $\operatorname{set}^{-1}(-\infty, \alpha)$. Since $x$ does not belong to the closed level $f^{-1}(\alpha)$, it has a connected open neighborhood $U$ that does not intersect with $f^{-1}(\alpha)$. Then $f(U)$ is a connected subset of $R$ that contains $f(x)<\alpha$ but not $\alpha$. Hence $f(U) \subset(-\infty, \alpha)$, i.e. $U \subset f^{-1}(-\infty, \alpha)$, and so $x$ is an interior point of the set $f^{-1}(-\infty, \alpha)$. This proves that $f^{-1}(-\infty, a)$ is open, and the openness of the set $f^{-1}(\alpha, \infty)$ is proved similarly. Thus $(a) \Rightarrow(b)$, and the implications $(\mathrm{b}) \Rightarrow$ (c) and (c) $\Rightarrow$ (a) are quite obvious.

2.2. THEOREM. The set $Y_{k}(f)$ is bilaterally closed.

Proof. If $\alpha$ is any bilateral limit point of the set $Y_{k}(f)$, there exist two sequences $\left\{\alpha_{n}\right\},\left\{\beta_{n}\right\}$ of distinct points of $Y_{k}(f)$ such that $\alpha_{n}$ increases to $\alpha$ and $\beta_{n}$ decreases to $\alpha$ as $n \rightarrow \infty$. According to Lemma 2.1 the set

$$
f^{-1}\left[\alpha_{n}, \beta_{n}\right]=\left\{f^{-1}\left(-\infty, \beta_{n}\right]\right\} \cap\left\{f^{-1}\left[\alpha_{n}, \infty\right)\right\}
$$

is closed for every $n$, and so the level

$$
f^{-1}(\alpha)=\bigcap_{n=1}^{\infty} f^{-1}\left[\alpha_{n}, \beta_{n}\right]
$$

is closed, i.e. $\alpha \in Y_{k}(f)$.

As an easy consequence of the above theorem we obtain

2.3. COROLLARY. The set $Y_{k}(f)$ contains every open interval in which it is dense, and it is further $a G_{\delta}$ set with a nowhere dense boundary.

2.4. Remark. As a converse of Theorem 2.2 it has been proved by Lipiński [11], Theorem 2, that for every bilaterally closed subset $E$ of $R$ there exists a connected function $f: R \rightarrow R$ such that $Y_{k}(f)=E$. It remains to investigate the validity of this converse for more general locally connected spaces $X$.

2.5. THEOREM. (a) If $\alpha \in R$ is a limit point of the set $Y_{k}(f)$ from below (above), then $f$ is lower (upper) semicontinuous at every point of the level $f^{-1}(\alpha)$.

(b) If $\alpha$ is a bilateral limit point of $Y_{k}(f)$, then $f$ is continuous at every point of the level $f^{-1}(\alpha)$.

(c) If $\alpha, \beta \in Y_{k}(f)$ and $\alpha<\beta$, then the oscillation of $f$ is not greater than $\beta-\alpha$ at any point of the inverse set $f^{-1}(\alpha, \beta)$. (d) The function $f$ is continuous relative to every set $A \subset X$ for which $\overline{f(A)}$ $\subset Y_{k}(f)$.

Proof. (a) Let first $\alpha$ be a limit point of $Y_{k}(f)$ from below and $x \in f^{-1}(\alpha)$. Given $\lambda<f(x)=\alpha$, we can find a point $\beta \in Y_{k}(f)$ such that $\lambda<\beta<\alpha$. Then the set $U=f^{-1}(\beta, \infty)$ is open by Lemma 2.1 , and clearly $x \in U$. At every point $y \in U$ we have $f(y)>\beta>\lambda$, and so $f$ is lower semicontinuous at $x$. The other part of (a) follows on applying the above to the function $-f$.

(b) This part follows on combining the two parts of (a).

(c) If $\alpha, \beta \in Y_{k}(f)$ and $\alpha<\beta$, then the set

$$
f^{-1}(\alpha, \beta)=\left\{f^{-1}(-\infty, \beta)\right\} \cap\left\{f^{-1}(\alpha, \infty)\right\}
$$

is open by Lemma 2.1, and so at any point of $f^{-1}(\alpha, \beta)$ the oscillation of $f$ cannot be greater than the diameter of $f\left\{f^{-1}(\alpha, \beta)\right\}$, which in turn cannot be greater than $\beta-\alpha$.

(d) Let $A$ be a subset of $X$ such that $\overline{f(A)} \subset Y_{k}(f)$, and let $g$ denote the restriction of $f$ to $A$. Putting $F=\overline{g(A)}$, we have $g(A) \subset F \subset Y_{k}(f)$. Let $x$ be any point of $A$, and let $\alpha=g(x)$. If $F$ is disjoint with the interval $(-\infty, \alpha)$, then $g$ is clearly lower semicontinuous at $x$. Otherwise let $\beta=\sup \{\gamma \in F: \gamma<\alpha\}$. Since $F$ is closed, we have $\beta \in F$ and $\beta \leqslant \alpha$. If $\beta=\alpha$, then $\alpha$ is a limit point of the set $Y_{k}(f)$ from below, and so $f$ is lower semicontinuous at $x$ by (a), whence $g$ is also lower semicontinuous at $x$. In case $\beta<\alpha$, then since $\beta \in F \subset Y_{k}(f)$, the set $U=f^{-1}(\beta, \infty)$ is open by Lemma 2.1; clearly $x \in U$ and at every point $y \in A \cap U$ we have $g(y)$ $\geqslant \alpha=g(x)$, whence $g$ is again lower semicontinuous at $x$. This proves the lower semicontinuity of $g$ at $x$. A similar argument yields its upper semicontinuity at $x$, and so $g$ is continuous at $x$.

Each of the parts (b) and (c) of Theorem 2.5 yields the following theorem of Lipiński [10]:

2.6. COROLLARY. If the set $Y_{k}(f)$ is dense in $R$ (or in the interior of the range of $f$ ), then $f$ is continuous.

2.7. Remark. Regarding the part (d) of Theorem 2.5 it may be observed that the function $f$ is not continuous in general relative to the set $S_{k}(f) \equiv f^{-1}\left\{Y_{k}(f)\right\}$. For let $X=R$ and $f(x)=x-1$ for $x<0, f(0)=1$ and $f(x)=\sin (1 / x)$ for $x>0$. It can be easily verified that $f$ is weakly connected and its restriction to the set $S_{k}(f)=(-\infty, 0) \cup f^{-1}(1)$ is discontinuous at 0 .

2.8. Definitron. Let a set in any topological space $X$ be called composed if all of its components are closed.

Let us observe here that every closed set is composed, and a composed set is in turn closed when it has only finitely many components; Also, the intersection, of an arbitrary family of composed sets is composed, but the union of two composed sets is not in general composed. In fact, as it is clear from the following 
two subsets of $R^{2}$, even the union of a composed set with a closed set need not be composed:

$$
\begin{aligned}
& A=\left\{(x, y): x=1, \frac{1}{2}, \frac{1}{3}, \ldots, 0 \leqslant y \leqslant 1\right\} \\
& B=\{(x, y): 0 \leqslant x \leqslant 1, y=0\} .
\end{aligned}
$$

2.9. THEOREM. Let: $X$ be any topological space, $Y$ a $T_{1}{ }^{- \text {space }}$ and $f: X \rightarrow Y$ be a connected function. If $C$ is any composed set in $Y$, then so is $f^{-1}(C)$.

Consequently, the levels of $f$ are composed and we have $Y_{c}(f) \subset Y_{k}(f)$.

Proof. Suppose there exists a composed set $C$ in $Y$ such that $f^{-1}(C)$ is not composed. Then $f^{-1}(C)$ has a component $A$ that is not closed. Let $x \in \bar{A}-A$. Since $A \cup\{x\}$ is connected, it follows that $x \notin f^{-1}(C)$ and that the set $f(A) \cup\{f(x)\}$ is connected. However, $f(A)$ being a connected subset of $C$, it is contained in some component $B$ of $C$, and since $C$ is composed, $B$ is closed. Thus $f(A)$ is contained in the closed set $B$, whereas $f(x) \notin B$. Since $\{f(x)\}$ is closed, this contradicts the connectedness of the set $f(A) \cup\{f(x)\}$

It follows from the above theorem that every connected weakly monotone function with a $T_{1}$ range has closed levels, strengthening thereby Theorem 3.1 of Pervin and Levine [13]. Although Theorem 2.9 is not altogether new (see Sanderson [14], Theorems 1, 3), it does provide in the above form a new property of continuous functions $f$ with $T_{1}$ range, viz. that $f^{-1}$ preserves composed sets. With the help of Corollary 2.6 it further gives

2.10. CoRollary. Let $X$ be a locally connected space and $f: X \rightarrow R$ be connected. If the set $Y_{c}(f)$ is dense in $R$, then $f$ is continuous.

In particular, $f$ is continuous whenever it is weakly monotone.

2.11. Corollary. If $f$ is connected and it is discontinuous at a dense set of points in $X$, then $f$ is nowhere weakly monotone.

Proof. Suppose $f$ is weakly monotone on some nonempty open set $U$ in $X$. The subspace $U$ is clearly locally connected. As a subset of $U$ is connected relative to $U$ if and only if it is connected in $X$, the restriction $g$ of $f$ to $U$ is also connected. Hence, by Corollary $2.10, g$ is continuous, which contradicts the hypothesis.

When the range $Y$ is a "semi-locally-connected" space, viz. every point of $Y$ has a local base of neighborhoods whose complements have only finitely many components, with the help of Theorems 8 and 9 of Sanderson [14] we further obtain from Theorem 2.9,

2.12. Corollary. If $X$ is any topological space and $Y$ is a semi-locally-connected $T_{1}$-space, then every connected monotone function $f: X \rightarrow Y$ is continuous.

\section{Connected levels and monotonicity.}

3.1. Definition. (a) A sequence $\left\{E_{n}\right\}$ of sets is said to be increasing (decreasing) if $E_{n} \subset E_{n+1}\left[\supset E_{n+1}\right.$ ] for every $n$. Let a topological space $X$ be called $\sigma$-coherent if the intersection of every decreasing sequence of closed connected sets in $X$ is connected. (b) Given two topological spaces $X$ and $Y$, a function $f: X \rightarrow Y$ is said to be proper [1] (or "compact" [19]) if the set $f^{-1}(K)$ is compact for every compact set $K$ in $Y$. Analogously, let $f$ be called relatively proper if $f^{-1}(K)$ is relatively compact for every relatively compact set $K$ in $Y$, or equivalently if $f^{-1}(K)$ is relatively compact for every compact set $K$ in $Y$.

It may be observed here that the space $R$ is $\sigma$-coherent, and so is every compact Hausdorff space $X$ (see [9], p. 170, Theorem 5). Also every proper function is relatively proper, and a continuous function with a Hausdorff range is proper whenever it is relatively proper. However, among discontinuous functions the class of relatively proper functions is much wider than that of proper functions. For a function with a compact domain is always relatively proper, but a function with compact Hausdorff domain and range is proper if and only if it is continuous.

From now on we return to our standard hypothesis that $f$ is a weakly connected real-valued function on a locally connected space $X$.

3.2. LemMa. Suppose $X$ and $f$ are connected.

(a) If $\alpha \in Y_{c}(f)$, then the sets $f^{-1}(-\infty, \alpha]$ and $f^{-1}[\alpha, \infty)$ are both closed and connected.

(b) If $\alpha, \beta \in Y_{c}(f)$ and $\alpha<\beta$, then the set $f^{-1}[\alpha, \beta]$ is also closed and connected.

Proof. (a) Since $f$ is connected, its connected level $f^{-1}(\alpha)$ is closed by Theorem 2.9. Hence the sets

$$
A=f^{-1}(-\infty, \alpha] \text { and } B=f^{-1}[\alpha, \infty)
$$

are both closed by Lemma 2.1. Since $A \cup B=X$ and $A \cap B=f^{-1}(\alpha)$ are both connected, it follows that $A$ and $B$ are also connected (see [9], p. 133).

(b) The connected levels $f^{-1}(\alpha)$ and $f^{-1}(\beta)$ are clearly closed, and so by Lemma 2.1 the sets

$$
A=f^{-1}(-\infty, \alpha] \quad \text { and } \quad C=f^{-1}[\alpha, \beta]
$$

are closed: Since $A \cup C=f^{-1}(-\infty, \beta]$ is connected by (a), and $A \cap C$ $=f^{-1}(\alpha)$ is also connected, it follows as above that $C$ is connected.

3.3. THEOREM. If $X$ and $f$ are connected, and either

(a) $X$ is $\sigma$-coherent, or

(b) $X$ is Hausdorff and $f$ is relatively proper,

then the set $Y_{c}(f)$ is bilaterally closed.

Proof. Let $\alpha$ be any bilateral limit point of $Y_{c}(f)$. Then there exist two sequences $\left\{\alpha_{n}\right\},\left\{\beta_{n}\right\}$ of distinct points of $Y_{c}(f)$ such that $\alpha_{n}$ increases to $\alpha$ and $\beta_{n}$ quences $\left\{\alpha_{n}\right\},\left\{\beta_{n}\right\}$ of decreases to $\alpha$ as $n \rightarrow \infty$. According to Lemma 3.2(b), the set $f^{-1}\left[\alpha_{n}, \beta_{n}\right]$ is closed decreases to $\alpha$ as $n \rightarrow \infty$. According to $f^{-1}\left[\alpha_{n}, \beta_{n}\right]$ decreases to $f^{-1}(\alpha)$ as $n \rightarrow \infty$, the level $f^{-1}(\alpha)$ is clearly connected when $X$ is $\sigma$-coherent.

In case (b), for each natural number $n$, the set $f^{-1}\left[\alpha_{n}, \beta_{n}\right]$ is further relatively 
compact; and since it is already closed and connected, it is in fact a continuum (viz. compact, connected and Hausdorff). Hence the level

$$
f^{-1}(\alpha)=\bigcap_{n=1}^{\infty} f^{-1}\left[\alpha_{n}, \beta_{n}\right]
$$

is also a continuum (see [9], p. 170, Theorem 5).

3.4. Remark. Corollary 2.3 is thus equally valid for the set $Y_{c}(f)$ when $f$ satisfies one of the hypotheses of Theorem 3.3. If $E$ is any bilaterally closed subset of $R$, then according to Theorem 1 of Lipiński [11] there exists a continuous function $f: R \rightarrow R$ for which $Y_{c}(f)=E$.

3.5. Lema. If $X$ and $f$ are connected and $\alpha \in R$ is a limit point of $Y_{c}(f)$ from below, then the set $f^{-1}(-\infty, \alpha)$ is open and its boundary is contained in the level $f^{-1}(\alpha)$.

Proof. There exists an increasing sequence $\left\{\alpha_{n}\right\}$ of elements of $Y_{c}(f)$ such that $\alpha_{n}$ converges to $\alpha$ as $n \rightarrow \infty$. For every natural number $n$, the level $f^{-1}\left(\alpha_{n}\right)$ is closed by Theorem 2.9 , and so the $\operatorname{set} f^{-1}\left(-\infty, \alpha_{n}\right)$ is open by Lemma 2.1. Hence the set

is open.

$$
f^{-1}(-\infty, \alpha)=\bigcup_{n=1}^{\infty} f^{-1}\left(-\infty, \alpha_{n}\right)
$$

According to Lemma 3.2 (a), the set $f^{-1}\left(-\infty, \alpha_{n}\right.$ ] is connected for every $n$ and as the sequence $\left\{f^{-1}\left(-\infty, \alpha_{n}\right]\right\}$ is increasing, it follows that its union $f^{-1}(-\infty, \alpha)$ is connected (see [9], p. 132). Thus $f^{-1}(-\infty, \alpha)$ is contained in some component $C$ of the set $f^{-1}(-\infty, \alpha]$. But the latter set is composed by Theorem 2.9 and so $C$ is closed. Hence we have

$$
\left\{f^{-1}(-\infty, \alpha)\right\}^{-} \subset C \subset f^{-1}(-\infty, \alpha] .
$$

As $f^{-1}(-\infty, \alpha)$ is open, it follows that the boundary of this set is contained in the level $f^{-1}(\alpha)$.

3.6. THEOREM. If $X$ and $f$ are connected, then $f$ is continuous relative to the closure of $S_{c}(f)$. Consequently, if the set $S_{c}(f)$ is further dense in $X$, then $f$ continuous.

Proof. Suppose $S=S_{c}(f)$ is nonempty, and let $g$ denote the restriction of $f$ to $\bar{S}$. As the intervals $(-\infty, \alpha)$ and $(\alpha, \infty), \alpha \in R$, form together a subbase of the topology of $R$, it is sufficient to show that the set $g^{-1}(-\infty, \alpha)$ is open relative to $\bar{S}$ for every $\alpha \in R$, for on applying this result to the function $-f$ is open relative to $S$ for every $\alpha \in R$, for on applying this result to the function $-f$ the same follows
for the set $g^{-1}(\alpha, \infty)$.

Given $\alpha \in R$, let

$$
\beta=\sup \left\{y \in Y_{c}(f): y \leqslant \alpha\right\} \quad \text { and } \quad \gamma=\inf \left\{y \in Y_{c}(f) ; y>\dot{\alpha}\right\} \text {. }
$$

Then $\beta \leqslant \alpha \leqslant \gamma$, and at least one of $\beta$ and $\gamma$ is finite. In case $\beta=\alpha$, then either $\alpha$ belongs to $Y_{c}(f)$ or it is a limit point of $Y_{c}(f)$ from below, and so by Lemmas 3.2 and 3.5 the set $f^{-1}(-\infty, \alpha)$ is open in each case, whence $g^{-1}(-\infty, \alpha)$ is open relative to its domain $\bar{S}$. So let $\beta<\alpha$.

When $\beta$ is finite, we claim that

$$
\left\{S \cap f^{-1}(-\infty, \beta]\right\}^{-} \subset f^{-1}(-\infty, \beta] .
$$

For if $\beta \in Y_{c}(f)$, then the set $f^{-1}(-\infty, \beta]$ is closed by Lemma 3.2, which implies (1), and if $\beta \notin Y_{c}(f)(\supset f(S))$, then $\beta$ is a limit point of $Y_{c}(f)$ from below, and so with the help of Lemma 3.5 we get

$$
\left\{S \cap f^{-1}(-\infty, \beta]\right\}^{-} \subset\left\{f^{-1}(-\infty, \beta)\right\}^{-} \subset f^{-1}(-\infty, \beta] .
$$

In case $\gamma$ is finite, it is proved similarly that

$$
\left\{S \cap f^{-1}[\gamma, \infty)\right\}^{-} \subset f^{-1}[\gamma, \infty) .
$$

When $\beta$ is finite and $\gamma=+\infty$, we have $f(S) \subset(-\infty, \beta]$, and so with the help of (1) we obtain

$$
\bar{S}=\left\{S \cap f^{-1}(-\infty, \beta]\right\}^{-} \subset f^{-1}(-\infty, \beta],
$$

proving thereby that $g^{-1}(-\infty, \alpha)=\bar{S}$. In case $\gamma$ is finite and $\beta=-\infty$, it follows similarly from $(2)$ that $g^{-1}(-\infty, \alpha)=\varnothing$.

Let finally $\beta$ and $\gamma$ be both finite. As the interval $(\beta, \gamma)$ does not contain any point of $f(S)$, we have

$$
S=\left\{S \cap f^{-1}(-\infty, \beta]\right\} \cup\left\{S \cap f^{-1}[\gamma, \infty)\right\},
$$

and so with the help of (1) and (2) we get

$$
\begin{aligned}
\bar{S} & =\left\{S \cap f^{-1}(-\infty, \beta]\right\}^{-} \cup\left\{S \cap f^{-1}[\gamma, \infty)\right\}^{-} \\
& \subset\left\{\bar{S} \cap f^{-1}(-\infty, \beta]\right\} \cup\left\{\bar{S} \cap f^{-1}[\gamma, \infty)\right\} \subset \bar{S} .
\end{aligned}
$$

Thus

$$
\bar{S}=\left\{\bar{S} \cap f^{-1}(-\infty, \beta]\right\} \cup\left\{\bar{S} \cap f^{-1}[\gamma, \infty)\right\},
$$

and as the two sets on the right of this equality are disjoint, we further obtain $\left\{S \cap f^{-1}(-\infty, \beta]\right\}^{-}=\bar{S} \cap f^{-1}(-\infty, \beta], \quad\left\{S \cap f^{-1}[\gamma, \infty)\right\}^{-}=\bar{S} \cap f^{-1}[\gamma, \infty)$, i.e. the two sets on the right of (3) are both closed. Hence

$$
g^{-1}(-\infty, \alpha)=\bar{S}-\left\{\bar{S} \cap f^{-1}[\gamma, \infty)\right\}
$$

is open relative to $\bar{S}$. This completes the proof of the theorem.

3.7. Proposition. If $X$ and $f$ are connected, then $f$ is monotone if and only if it is weakly monotone.

Proof. The necessity part of the proposition is obvious. To prove the sufficiency, suppose $f$ is weakly monotone, i.e. $Y_{c}(f)=R$. A subset of $R$ is connected if and only if it is a subinterval $I$ of $R$. If $I$ is a degenerate interval, the set $f^{-1}(I)$ is obvi- 
ously connected. When $I$ is a non-degenerate interval, finite or infinite, it can always be expressed as the union of an increasing sequence $\left\{I_{n}\right\}$ of finite closed intervals. Then we have

$$
f^{-1}(I)=\bigcup_{n=1}^{\infty} f^{-1}\left(I_{n}\right)
$$

where, by Lemma $3.2\left(\right.$ b), the set $f^{-1}\left(I_{n}\right)$ is connected for each $n$. Since $\left\{f^{-1}\left(I_{n}\right)\right\}$ is an increasing sequence of connected sets, it follows that $f^{-1}(I)$ is connected. This proves that $f$ is monotone.

3.8. COROLLARY. If $f$ is connected and nowhere monotone, then it is also nowhere weakly monotone.

Proof. Suppose $f$ is weakly monotone on some nonempty open subset $U$ of $X$. Let $V$ be any component of $U$, and let $g$ and $h$ denote the restrictions of $f$ to the sets $U$ and $V$ respectively. As a subset of $V$ is connected with respect to the relative topology of $V$ if and only if it is connected in $X$, the function $h$ is equally connected. Further, given $\alpha \in R$, as the function $g$ is weakly monotone, its level $g^{-1}(\alpha)$ is a connected subset of $U$. If $g^{-1}(\alpha)$ intersects with $V$, then clearly $g^{-1}(\alpha) \subset V$, so that $h^{-1}(\alpha)=g^{-1}(\alpha)$ is connected, and if $g^{-1}(\alpha)$ is disjoint with $V$, then $h^{-1}(\alpha)=\varnothing$, which is again connected. Hence the function $h$ is weakly monotone. Since $V$ is a connected open subspace of $X$, it is also locally connected (see [9], p. 230), and so it follows from the above proposition that $h$ is monotone. This contradicts the hypothesis of nowhere monotonicity of $f$.

3.9. THEOREM. Suppose $X$ is connected and $\sigma$-coherent and $f$ is conniected. If either the set $Y_{c}(f)$ is dense in $R$ (or in the interior of the range of $f$ ), or the set $S_{c}(f)$ is dense in $X$, then the function $f$ is continuous and monotone.

Proof. According to Corollary 2.10 and Proposition 3.7, it suffices to show that $f$ is weakly monotone.

When the set $S_{c}(f)$ is dense in $X$, the function $f$ is continuous by Theorem 3.6, and so we have

$$
f(X)=f\left(\overline{S_{c}(f)}\right) \subset \overline{f\left(S_{c}(f)\right)} \subset \overline{Y_{c}(f)} .
$$

Since the level $f^{-1}(\alpha)$ is trivially connected when $\alpha$ is not in the range of $f$, it follows that the set $Y_{c}(f)$ is dense in $R$.

When, on the other hand, $Y_{c}(f)$ is dense in $R$, it follows from Theorem 3.3 that $f$ is weakly monotone.

As for the role of connectedness of $f$ in the above theorem, it may be observed that there exist injective functions $f: R \rightarrow R$ that are not even Lebesgue measurable (see Sierpiński [15]), and so are clearly neither continuous nor monotone.

3.10. THEOREM. Suppose $X$ is connected and Hausdorff, and that $f$ is connected and relatively proper. If either the set $Y_{c}(f)$ is dense in $R$, or the set $S_{c}(f)$ is dense in $X$, then the function $f$ is continuous, monotone, Morrey monotone arid proper.

Proof. In either case the continuity and monotonicity of $f$ follow exactly as above. If further $K$ is any compact subset of $R$, then since $f$ is continuous and relatively proper, the set $f^{-1}(K)$ is a closed subset of some compact set, and so is compact. Hence $f$ is proper. In particular, the levels of $f$ are compact, and so $f$ is equally Morrey monotone.

3.11. Problem. A connected function $f: R \rightarrow R$ was proved in Theorem 2 of [4] to be always monotone (in the usual sense) relative to the set $\overline{S_{c}(f)}$. It remains to investigate under what hypotheses on $X$ and $f$ a connected function $f: X \rightarrow R$ is monotone, or weakly monotone, relative to the set $\overline{S_{c}(f)}$ in general. For a partial solution see Proposition 4.7.

4. Singleton levels and injectivity. Let $\partial E$ denote the boundary of any set $E \subset X$.

4.1. LEMMA. If $\alpha \in Y_{k}(f)$, then

$$
\partial f^{-1}(\alpha)=\partial f^{-1}(-\infty, \alpha) \cup \partial f^{-1}(\alpha, \infty) .
$$

Proof. As the level $f^{-1}(\alpha)$ is closed, according to Lemma $2.1, f^{-1}(-\infty, \alpha)$ is an open subset of the closed set $f^{-1}(-\infty, \alpha]$. Hence $f^{-1}(\alpha)$ contains the boundary of $f^{-1}(-\infty, \alpha)$, and as the interior of $f^{-1}(\alpha)$ cannot contain any limit point of $f^{-1}(-\infty, \alpha)$, it follows that $\partial f^{-1}(-\infty, \alpha) \subset \partial f^{-1}(\alpha)$. By a similar argument we obtain $\partial f^{-1}(\alpha, \infty) \subset \partial f^{-1}(\alpha)$. Thus we have

$$
\begin{aligned}
\partial f^{-1}(\alpha) & =\partial\left\{f^{-1}(-\infty, \alpha) \cup f^{-1}(\alpha, \infty)\right\} \\
& \subset \partial f^{-1}(-\infty, \alpha) \cup \partial f^{-1}(\alpha, \infty) \subset \partial f^{-1}(\alpha),
\end{aligned}
$$

which establishes the required equality.

4.2. LeMMA. Suppose $X$ is coninected and $\alpha \in Y_{k}(f)$.

(a) If the boundaries of the sets $f^{-1}(-\infty, \alpha)$ and $f^{-1}(\alpha, \infty)$ are connected, then the level $f^{-1}(\alpha)$ is also connected.

(b) If the level $f^{-1}(\alpha)$ is a boundary set, then the boundaries of the sets $f^{-1}(-\infty, \alpha)$ and $f^{-1}(\alpha, \infty)$ are not disjoint.

Proof. (a) Suppose the boundaries $\partial f^{-1}(-\infty, \alpha)$ and $\partial f^{-1}(\alpha, \infty)$ are connected but the level $f^{-1}(\alpha)$ is not connected. Then since $f^{-1}(\alpha)$ is closed, it can be separated into two nonempty disjoint closed sets $F_{1}$ and $F_{2}$. As the connected sets $\partial f^{-1}(-\infty, \alpha)$ and $\partial f^{-1}(\alpha, \infty)$ are, by Lemma 4.1 , both contained in $f^{-1}(\alpha)$ $=F_{1} \cup F_{2}$, each of them is contained in one of $F_{1}$ and $F_{2}$. If both are contained in the same set, say $F_{1}$, then the set

$$
F=f^{-1}(-\infty, \alpha) \cup f^{-1}(\alpha, \infty) \cup F_{1}
$$

is closed, and so $X=F \cup F_{2}$ provides a separation of $X$, contradicting thereby the connectedness of $X$. In case the two boundaries are contained in different sets, say $\partial f^{-1}(-\infty, \alpha) \subset F_{1}$ and $\partial f^{-1}(\alpha, \infty) \subset F_{2}$, then

$$
X=\left\{f^{-1}(-\infty, \alpha) \cup F_{1}\right\} \cup\left\{f^{-1}(\alpha, \infty) \cup F_{2}\right\}
$$

provides a separation of $X$, which is again a contradiction. Hence the level $f^{-1}(\alpha)$ is connected. 
(b) If the closed level $f^{-1}(\alpha)$ is a boundary set, then $\partial f^{-1}(\alpha)=f^{-1}(\alpha)$, and so with the help of Lemma 4.1 we have

$$
f^{-1}(\alpha)=\partial f^{-1}(-\infty, \alpha) \cup \partial f^{-1}(\alpha, \infty) .
$$

If the two boundaries on the right are disjoint, then

$$
X=\left\{f^{-1}(-\infty, \alpha)\right\}^{-} \cup\left\{f^{-1}(\alpha, \infty)\right\}^{-}
$$

provides a separation of $X$. Hence the two boundaries cannot be disjoint.

4.3. LemMa. Suppose $X$ is connected and Hausdorff.

(a) If $\alpha \in R$ is a limit point of $Y_{1}(f)$ from below and $f^{-1}(-\infty, \alpha) \neq X$, then the boundary of the set $f^{-1}(-\infty, \alpha)$ is singleton.

(b) If $\alpha$ is a bilateral limit point of $Y_{1}(f)$, then the level $f^{-1}(\alpha)$ is closed and connected, and if it is further a boundary set, then it is also singleton.

Proof. (a) Since $X$ is Hausdorff, every singleton level of $f$ is closed, and so it follows from Lemma 2.1, as in the proof of Lemma 3.5, that the set $U=f^{-1}(-\infty, \alpha)$ is open. As $U$ is by hypothesis a proper subset of the connected space $X$, it cannot be closed. Hence $\partial U \neq \varnothing$.

Suppose $\partial U$ contains two distinct points $x_{1}$ and $x_{2}$. As $\partial U$ is clearly disjoint with $U$, we have $f\left(x_{i}\right) \geqslant \alpha$ for $i=1,2$. The space $X$ being Hausdorff and locally connected, the points $x_{1}, x_{2}$ have two disjoint connected open neighborhoods $V_{1}$ and $V_{2}$ respectively, and since $x_{1}, x_{2}$ are limit points of $U$, there exist two points $y_{1}, y_{2}$ in $U$ such that $y_{i} \in V_{i}(i=1,2)$. Thus $f\left(y_{i}\right)<\alpha$ for each $i$. Let

$$
\beta=\max \left\{f\left(y_{i}\right): i=1,2\right\} \text {. }
$$

Clearly $\beta<\alpha$, and so according to the hypothesis we can choose a point $\gamma$ in the set $Y_{1}(f)$ such that $\beta<\gamma<\alpha$. As the sets $f\left(V_{1}\right)$ and $f\left(V_{2}\right)$ are connected, we get

$$
\gamma \in(\beta, \alpha) \subset\left[f\left(y_{i}\right), f\left(x_{i}\right)\right] \subset f\left(V_{i}\right) \quad(i=1,2) .
$$

Thus the singleton level $f^{-1}(\gamma)$ meets two disjoint sets $V_{1}$ and $V_{2}$, which is not possible. Hence $\partial U$ is singleton.

(b) Let $\alpha$ be a bilateral limit point of the set $Y_{1}(f)$. Since $Y_{1}(f) \subset Y_{k}(f)$, it follows from Theorem 2.2 that the level $f^{-1}(\alpha)$ is closed. As the sets $\partial f^{-1}(-\infty, \alpha)$ and $\partial f^{-1}(\alpha, \infty)$ are singleton by above, it follows from Lemma 4.2 (a) that the level $f^{-1}(\alpha)$ is connected.

If the closed level $f^{-1}(\alpha)$ is further a boundary set, then clearly $\partial f^{-1}(\alpha)=f^{-1}(\alpha)$, and so with the help of Lemma 4.1 we have

$$
f^{-1}(\alpha)=\partial f^{-1}(-\infty, \alpha) \cup \partial f^{-1}(\alpha, \infty) .
$$

According to Lemma 4.2(b) the two boundaries on the right must intersect, and as they are both singleton, it follows that $f^{-1}(\alpha)$ is singleton.

4.4. THEOREM. Suppose $X$ is connected and Hausdorff. (a) If $X$ is separable, then the set $Y_{1}(f)$ is of the form $F-C$, where $F$ is closed and $C$ is countable.

(b) If $f$ is nowhere constant, then $Y_{1}(f)$ is bilaterally closed.

Proof. (a) Let $A$ denote the set of values of $\alpha$ in $R$ for which the level $f^{-1}(\alpha)$ has a nonempty interior. As the levels of $f$ are mutually disjoint, the set $A$ is clearly countable when $X$ is separable. According to Lemma 4.3(b), the set $Y_{1}(f)$ contains all of its bilateral limit points which are outside $A$. As the set $B$ of unilateral limit points of $Y_{1}(f)$ is also countable, we have $Y_{1}(f)=\overline{Y_{1}\left(f^{\prime}\right)}-C$, where $C$ is a subset of $\boldsymbol{A} \cup B$, and so is countable.

(b) When $f$ is nowhere constant, each of its levels is a boundary set, and so in this case, by Lemma $4.3(\mathrm{~b})$, the set $Y_{1}(f)$ contains all of its bilateral limit points.

4.5. Remark. It was proved originally by Sierpiński [16] that for every function $f: R \rightarrow R$ with a closed graph the set $Y_{1}(f)$ is $G_{\delta}$. Under the hypothesis of Theorem 4.4, part (a), the set $Y_{1}(f)$ is clearly $G_{\delta}$, and further if $Y_{1}(f)$ is dense in some subinterval $I$ of $R$, then it contains all but a countable set of points of $I$. Moreover, if $E$ is any subset of $R$ of the form $F-C$, then according to Theorem 3 of Lipiński [11] there exists a continuous function $f: R \rightarrow R$ for which $Y_{1}(f)=E$.

4.6. THEOREM. If $X$ is connected and Hausdorff, and $f$ assumes a dense set of its values only once, then $f$ is continuous and monotone. In case $f$ is further nowhere constant, then it is also injective.

Proof. Since $X$ is Hausdorff, we have $Y_{1}(f) \subset Y_{k}(f)$, and so the function $f$ is continuous by Corollary 2.6. We shall assume that $f$ is not constant, for the result holds trivially otherwise. Then, $X$ being connected, the range of $f$ is a non-degenerate subinterval of $R$.

When $\alpha$ is an interior point of $f(X)$, it is clearly a bilateral limit point of $Y_{1}(f)$, and so the level $f^{-1}(\alpha)$ is connected by Lemma 4.3(b). If $\alpha \in f(X)$ is not in the interior of $f(X)$, then it must be an endpoint of the interval $f(X)$. Suppose $\alpha=\max \{f(x): x \in X\}$. Then the set $f^{-1}(\alpha, \infty)$ is empty, whence $\partial f^{-1}(\alpha, \infty)=\varnothing$, and since $\alpha$ is a limit point of $Y_{1}(f)$ from below, it follows from Lemma 4.3(a) that $\partial f^{-1}(-\infty, \alpha)$ is singleton. Thus both the boundaries $\partial f^{-1}(-\infty, \alpha)$ and $\partial f^{-1}(\alpha, \infty)$ are connected, and so the level $f^{-1}(\alpha)$, which is clearly closed, is further connected by Lemma 4.2(a). A similar proof holds when $\alpha=\min \{f(x): x \in X\}$. This proves that the function $f$ is weakly monotone, and its monotonicity now follows from Proposition 3.7.

If $f$ is further nowhere constant, then according to Theorem 4.4(b) the set $Y_{1}(f)$ contains all the interior points of the interval $f(X)$. When $\alpha=\max \{f(x): x \in X\}$, then, as seen above, we have $\partial f^{-1}(\alpha, \infty)=\varnothing$ and the boundary $\partial f^{-1}(-\infty, \alpha)$ is singleton. As the level $f^{-1}(\alpha)$ is now a closed boundary set, we have $\partial f^{-1}(\alpha)$ $=f^{-1}(\alpha)$. Hence, with the help of Lemma 4.1 we get $f^{-1}(\alpha)=\partial f^{-1}(-\infty, \alpha)$ which is singleton. As the above arguments remain valid when $\alpha=\min \{f(x): x \in X\}$, this proves that $f$ is injective. 
4.7. Proposition. If $X$ is connected and Hausdorff, and $f$ is connected, then $f$ is weakly monotone relative to the set $S_{c}(f) \cup \overline{S_{1}(f)}$. Moreover, if $f$ is further nowhere constant, then it is injective on the closure of $S_{1}(f)$.

Proof. Let us denote the sets $S_{c}(f), S_{1}(f), Y_{c}(f)$ and $Y_{1}(f)$ simply by $S_{c}, S_{1}, Y_{c}$ and $Y_{1}$ respectively. Let, further, $A=S_{c} \cup \overline{S_{1}}$. Since $S_{1} \subset S_{c}$, and the function $f$ is continuous relative to $\overline{S_{c}}$ (see Theorem 3.6), we have

$$
f\left(\overline{S_{1}}\right) \subset \overline{f\left(S_{1}\right)}=\overline{Y_{1}}, \quad f(A) \subset Y_{c} \cup \overline{Y_{1}} .
$$

When $\alpha \in Y_{c}$, the level $f^{-1}(\alpha)$ is a connected subset of $A$, and so it is equally connected with respect to the relative topology of $A$. So let $\alpha \in f(A)-Y_{c}$. Since $Y_{1} \subset Y_{c}, \alpha \notin Y_{1}$, and so, by (1), $\alpha$ is a limit point of $Y_{1}$. Also, by Lemma 4.3(b) every bilateral limit point of $Y_{1}$ is in $Y_{c}$. Hence $\alpha$ is a limit point of $Y_{1}$ from one and only one side, say from below. Clearly,

$$
A \cap f^{-1}(\alpha)=\overline{S_{1}} \cap f^{-1}(\alpha)
$$

and as $\alpha \notin Y_{1}$, we have

$$
\overline{S_{1}}=\left\{S_{1} \cap f^{-1}(-\infty, \alpha)\right\}^{-} \cup\left\{S_{1} \cap f^{-1}(\alpha, \infty)\right\}^{-} .
$$

According to Lemma 4.3(a), the set $f^{-1}(-\infty, \alpha)$ has a singleton boundary, and so the level $f^{-1}(\alpha)$ cannot contain more than one point of the first set on the right of (3). Further, since $\alpha$ is not a limit point of $Y_{1}$ from above, we have

$$
\beta \equiv \inf \left\{y \in Y_{1}: y>\alpha\right\}>\alpha .
$$

When $\beta=\infty$, the second set on the right of (3) becomes empty. Suppose $\beta<\infty$. If $\beta \in Y_{1}$, then the set $f^{-1}[\beta, \infty)$ is closed; otherwise $\beta$ is a limit point of $Y_{1}$ from above, and so according to Lemma 3.5 we have $\left\{f^{-1}(\beta, \infty)\right\}^{-} \subset f^{-1}[\beta, \infty)$. In any case we obtain

$$
\left\{S_{1} \cap f^{-1}(\alpha, \infty)\right\}^{-} \subset f^{-1}[\beta, \infty),
$$

and so the level $f^{-1}(\alpha)$ does not contain any point of the second set on the right of (3). Hence $f$ assumes $\alpha$ at most once on $\overline{S_{1}}$, and so at most once on $A$ by (2). This proves the weak monotonicity of $f$ relative to $A$.

Now suppose $f$ is nowhere constant, and let $\alpha \in f\left(\overline{S_{1}}\right)$. According to (1), $\alpha \in \overline{Y_{1}}$. The level $f^{-1}(\alpha)$ is clearly singleton when $\alpha \in Y_{1}$, and in the present case also when $\alpha$ is a bilateral limit point of $Y_{1}$ (see Lemma 4.3(b)). When $\alpha$ is not in $Y_{1}$ and is a. limit point of $Y_{1}$ only from one side, we have seen above that $f$ assumes $\alpha$ at most once on $\overline{S_{1}}$. Hence in this case $f$ is injective on $\overline{S_{1}}$.

4.8. THEOREM. If $X$ is connected and Hausdorff, $f$ is connected and the set $S_{1}(f)$ is dense in $X$, then $f$ is continuous, monotone and injective.

Proof. The function $f$ is clearly continuous by Theorem 3.6. We may assume that $X$ contains more than one point, for the result holds trivially otherwise. Then since $X$ is connected, it cannot have any isolated point, and so, under the present hypothesis, the function $f$ becomes nowhere constant. It now follows from Proposition 4.7 that $f$ is injective, and then from Proposition 3.7 that $f$ is monotone.

4.9. Remark. Tin jesults $4.4,4.6$ and 4.7 remain valid on replacing the hypothesis "nowhere constant" by "light". For each of these results is trivially valid when $X$ is empty or singleton, and when $X$ contains more than one point, then since $X$ is locally connected and connected, every light function on $X$ is nowhere constant.

5. Dense-in-itself levels and nowhere monotonicity. Let us recall that a topological space is said to be second countable if it has a countable base of open sets.

5.1. THEOREM. If $X$ is Hausdorff and second countable, then the sets $Y_{d}(f)$. and $Y_{p}(f)$ are both of the form $G_{\delta} \cup C$, where $C$ is countable.

Moreover, if $f$ is further nowhere constant, then the two sets are also of the form $F-M$, where $F$ is closed and $M$ is a meager $G_{\delta \sigma}$ set.

Proof. Since $X$ is second countable and locally connected, it has a countable base $\left\{U_{n}\right\}$ of connected open sets. Let $f_{n}$ denote, for each $n$, the restriction of $f$ to the set $U_{n}$. Given $\alpha \in R$, the level $f^{-1}(\alpha)$ has an isolated point if and only if $\alpha \in Y_{1}\left(f_{n}\right)$ for some $n$. Hence we have

$$
Y_{d}(f)=R-\bigcup_{n=1}^{\infty} Y_{1}\left(f_{n}\right)
$$

For every natural number $n$, the subspace $U_{n}$ is clearly connected, locally connected, Hausdorff and second countable. Also, a subset of $U_{n}$ is connected or open relative to $U_{n}$ if and only if it is so relative to $X$, and so the function $f_{n}$ is weakly connected. Hence, by Theorem 4.4(a), we have

$$
Y_{1}\left(f_{n}\right)=F_{n}-C_{n},
$$

where the set $F_{n}$ is closed relative to $U_{n}$ and $C_{n}$ is countable. Since $F_{n}$ is clearly an $F_{\sigma}$ set in $X$, with the help of $(*)$ we get

$$
Y_{d}(f)=R-\bigcup_{n=1}^{\infty}\left(F_{n}-C_{n}\right)=R-\left(F_{\sigma}^{\prime}-C\right)=G_{\delta} \cup C,
$$

where $C \subset \bigcup_{n=1}^{\infty} C_{n}$, and so is countable. Also, as the set $Y_{k}(f)$ is $G_{\delta}$ by Corollary 2.3, we further have

$$
Y_{p}(f)=Y_{k}(f) \cap Y_{d}(f)=G_{\delta} \cap\left(G_{\delta} \cup C\right)=G_{\delta} \cup C_{0},
$$

where $C_{0} \subset C$, and so is countable.

In case $f$ is nowhere constant, so is its restriction $f_{n}$ for each $n$, and so according Theorem 4.4 , part (b), the set $Y_{1}\left(f_{n}\right)$ is bilaterally closed. The set $A_{n}=Y_{1}\left(f_{n}\right)$ is thus a $G_{\delta}$ set with a nowhere dense boundary, and the set $B_{n}=A_{n}-A_{n}^{0}$ is in 
turn a nowhere dense $G_{\delta}$ set. Since $A_{n}=A_{n}^{0} \cup B_{n}$, once again with the help of (*) we get

$$
Y_{d}(f)=R-\bigcup_{n=1}^{\infty}\left(A_{n}^{0} \cup B_{n}\right)=R-(G \cup M)=F-M,
$$

where $G=\bigcup_{n=1}^{\infty} A_{n}^{0}$ is open, $F=R-G$ is closed and $M=\bigcup_{n=1}^{\infty} B_{n}$ is a meager $G_{\delta \sigma}$ set. Moreover,

$$
Y_{p}(f)=Y_{k}(f) \cap Y_{d}(f)=G_{\delta} \cap(F-M)=E-M,
$$

where $E=G_{\delta} \cap F$ is a $G_{\delta}$ set, and so is residual in the closure of $E$. Thus the $F_{\sigma}$ set $N=\bar{E}-E$ is meager relative to $\bar{E}$, and so in $X$, and we have

$$
Y_{p}(f)=(\bar{E}-N)-M=\bar{E}-(M \cup N),
$$

where $M \cup N$ is again a meager $G_{\delta \sigma}$ set.

Recalling that a set in $X$ is $\aleph_{1}$-dense if its intersection with every nonempty open set is uncountable, we obtain

5.2. COROLlary. If $X$ is Hausdorff and second countable, then each of the sets $Y_{d}(f)$ and $Y_{p}(f)$ is residual in every interval in which it is $\aleph_{1}$-dense, and if $f$ is further nowhere constant, then these sets are residual even in the intervals in which they are dense.

5.3. Remark. It was proved originally by Sierpiński [16] that for every function $f: R \rightarrow R$ with a closed graph the set $Y_{p}(f)$ is $F_{\sigma \delta}$. If on the other hand $E$ is any subset of $R$ of the form $G_{\delta} \cup C$, it has been proved by Lipiński [11], Theorem 4, that there exists a continuous function $f: R \rightarrow R$ for which $Y_{p}(f)=Y_{d}(f)=E$. Once again, it remains to investigate the validity of this converse of Theorem 5.1 for more general locally connected spaces $X$, and the same applies to the converse of Theorems 3.3 and 4.4 .

As for the nowhere monotonicity of a function $f$ in terms of its dense-initself levels, it is clear that $f$ is nowhere injective when the set $S_{d}(f)$ is dense in $X$ and if $f$ is further light, then it is even nowhere weakly monotone (for $f$ is injective if and only if it is light and weakly monotone). In case the set $Y(f)$ is dense in $R$, or equivalently if it is dense in the interior of the range of $f$, we still have the following

5.4. Proposition. If $X$ is dense-in-itself and the set $Y_{d}(f)$ is dense in $R$, then $f$ is nowhere injective. In case $f$ is further light, then it is also nowhere weakly monotone.

Proof. Suppose there exists a nonempty open set $U$ in $X$ such that $f$ is injective on $X$. Then $U$ contains a nonempty connected open set $V$, and $f$ is equally weakly connected and injective on $V$. As the space $X$ is dense-in-itself, the set $V$ contains more than one point, and so $f$ is not constant on $V$. It follows that the set $f(V)$ is a non-degenerate subinterval of $R$. If $\alpha \in f(V)$, then the level $f^{-1}(\alpha)$ contains one and only one point of $V$, and so it cannot be dense-in-itself. This contradicts the hypothesis that the set $Y_{d}(f)$ is dense in $R$. Hence the function $f$ is nowhere injective. The second part of the proposition is again an obvious consequence of the first.

5.5. THEOREM. Suppose $X$ is Hausdorff and second countable. If $f$ is either

(a) nowhere monotone, or

(b) nowhere constant and nowhere injective,

then the level $f^{-1}(\alpha)$ is a boundary set for every $\alpha \in R$ and it is dense-in-itself for a residual set of values of $\alpha$ in $R$.

Proof. Let the function $f$ satisfy one of the hypotheses (a) and (b). As the space $X$ is locally connected, in either case $f$ is nowhere constant. Consequently, every level of $f$ is a boundary set.

Let $\left\{U_{n}\right\}$ be a countable base of connected open sets in $X$, and let, for each $n$, $f_{n}$ denote the restriction of $f$ to $U_{n}$. Then we have, as before,

$$
Y_{d}(f)=R-\bigcup_{n=1}^{\infty} Y_{1}\left(f_{n}\right),
$$

and so it suffices to show that the set $Y_{1}\left(f_{n}\right)$ is nowhere dense in $R$ for each $n$.

Suppose there exists an $n$ for which the set $Y_{1}\left(f_{n}\right)$ is not nowhere dense. Then we can find two points $\alpha, \beta$ in $Y_{1}\left(f_{n}\right)$ such that $\alpha<\beta$ and the set $Y_{1}\left(f_{n}\right)$ is dense in $(\alpha, \beta)$. The set $f_{n}^{-1}(\alpha, \beta)$ is then open by Lemma 2.1 , and as this set is clearly nonempty, it contains a nonempty connected open set $V$. Let $g$ denote the restriction of $f_{n}$ to $V$. Since $f$ is nowhere constant, the range of $g$ is a non-degenerate subinterval of $(\alpha, \beta)$, and since $Y_{1}\left(f_{n}\right) \cap g(V) \subset Y_{1}(g)$, the set $Y_{1}(g)$ is dense in the range of $g$. As the subspace $V$ is clearly connected, locally connected and Hausdorff, and the function $g$ is weakly connected and nowhere constant, it follows from Therem 4.6 that $g$ is monotone and injective. This contradicts each of the hypotheses (a) and (b), and the theorem is thus proved.

As a continuous function has all of its levels closed, the above theorem yields the following generalization of the necessity part of Theorem 1 of [3]:

5.6. Corollary. Suppose $X$ is Hausdorff and second countable, and that $f$ is continuous. If $f$ is either. (a) nowhere monotone, or (b) nowhere constant and nowhere injective, then the level $f^{-1}(\alpha)$ is nowhere dense for every $\alpha \in R$ and it is perfect for a residual set of values of $\alpha$ in $R$.

A subset of $X$ is said to be scattered [8] if it does not contain any nonempty dense-in-itself set. If $P$ is any property of functions, a function $f$ is said to be intrinsically $P$ [5] if every nonempty open set in its domain contains a nonempty open set on which $f$ possesses $P$. As another easy consequence of Theorem 5.5 we have

5.7. Corollary. If $X$ is Hausdorff and second countable, and the level $f^{-1}(\alpha)$ is scattered for a residual set of values of $\alpha$ in $R$, then the function $f$ is intrinsically monotone. In case $f$ is further nowhere constant, then it is also intrinsically injective.

5.8. Remark. As it is clear from Proposition 5.4, the converse of the part (b) of Theorem 5.5 holds in general. The converse of its part (a) is also valid when 3 - Fundamenta Mathematicae Xcrur 
$X=R$ (for then $f$ is light if and only if it is nowhere constant), but not in general. For a counterexample consider the function $f(x, y)=x^{2}+y^{2}$ on $R^{2}$. It may be observed further that the hypothesis "nowhere constant" cannot be deleted from the part (b) of Theorem 5.5, for the Cantor's singular function is continuous and nowhere injective, but all but a countable set of its levels are singleton.

5.9. THEOREM. Suppose $X$ is Hausdorff and second countable, and that $f$ is connected. If $f$ is either (a) nowhere monotone, or (b) nowhere injective, then there exists a residual set of points $x$ in $X$ such that $x$ is a limit point of the level $f^{-1}\{f(x)\}$.

Proof. Let $\left\{U_{n}\right\}$ be a countable base of connected open sets in $X$, and for each $n$ let $f_{n}$ denote the restriction of $f$ to $U_{n}$. If $E$ denotes the set of points $x$ in $X$ such that $x$ is not a limit point of the level $f^{-1}\{f(x)\}$, it is clear that

$$
E=\bigcup_{n=1}^{\infty} S_{1}\left(f_{n}\right)
$$

It is sufficient to show that for each $n$ the set $S_{1}\left(f_{n}\right)$ is nowhere dense in $U_{n}$, for then $S_{1}\left(f_{n}\right)$ would be equally nowhere dense in $X$, and so $E$ would be meager in $X$.

Suppose there exists a natural number $n$ for which the set $S_{1}\left(f_{n}\right)$ is not nowhere dense in $U_{n}$. Then there exists a nonempty connected open set $V$ in $U_{n}$ such that $S_{1}\left(f_{n}\right)$ is dense in $V$. Denoting by $g$ the restriction of $f$ to $V$, we have $V \cap S_{1}\left(f_{n}\right)$ $\subset S_{1}(g)$, and so $S_{1}(g)$ is dense in $V$. Hence it follows from Theorem 4.8 that $g$ is monotone and injective. As this contradicts each of the hypotheses (a) and (b), the theorem is proved.

As an injective function is clearly Morrey monotone, the results 5.5, 5.6 and 5.9 are equally valid for nowhere Morrey monotone functions that are nowhere constant.

5.10. Problem. If a continuous real-valued function $f$ defined on a locally connected, separable, complete metric space $X$ (or on $R^{n}$ ) is nowhere monotone, does there exist a residual set of points $x$ in $X$ such that $x$ is a limit point of the level $f^{-1}\{f(x)\}$ along every simple arc in $X$ that has $x$ as an endpoint? The answer is known to be affirmative for $X=R$ (see [2], Theorem 2).

5.11. Definition. A topological space $X$ is said to be cyclicly connected (see Whyburn [18], p. 77) if every pair of points in $X$ is contained in some simple closed curve of $X$. Let $X$ be called further locally cyclicly connected if for each point $x$ in $X$ every open neighborhood of $x$ contains a cyclicly connected neighborhood of $x$,

It is clear that every locally cyclicly connected space is locally connected, and that the Euclidean space $R^{n}$ is locally cyclicly connected if and only if $n>1$. In case of locally cyclicly connected spaces Proposition 5.4 can be generalized as follows:

5.12. Proposition. If $X$ is dense-in-itself and locally cyclicly connected, then every connected function $f: X \rightarrow R$ is nowhere injective, and in case $f$ is further light, then it is also nowhere weakly monotone.

Proof. Given a nonempty open subset $U$ of $X$, let $x \in U$. There exists a cyclicly" connected neighborhood $V$ of $x$ such that $V \subset U$. As $X$ is dense-in-itself, there further exists a point $y$ in $V$ that is distinct from $x$. Suppose $f(y) \neq f(x)$, for otherwise $f$ is clearly not injective on $U$. Since $V$ is cyclicly connected, it contains a simple closed curve $C$ such that $x, y \in C$. Then $C=A \cup B$, where $A$ and $B$ are two simple arcs from $x$ to $y$ with no other common point. As the sets $A$ and $B$ are connected, so are their images $f(A)$ and $f(B)$, and so the value $\{f(x)+f(y)\} / 2$ is assumed by $f$ at two distinct points of $C$, one in $A$ and one in $B$. This proves that $f$ is nowhere injective, and the second part of the proposition follows from it without difficulty.

Theorems 5.5 and 5.9 assume in turn the following stregthened form:

5.13. Proposition. Suppose $X$ is locally cyclicly connected and second countable. If $f: X \rightarrow R$ is connected, then

(a) the level $f^{-1}(\alpha)$ is a dense-in-itself boundary set for all but countably many values of $\alpha$ in $R$, and

(b) for all but a countable set of points in $X$, every point $x$ is a limit point of the level $f^{-1}\{f(x)\}$.

In particular, if $f$ is continuous then all but countably many of its levels are nowhere dense and perfect.

Proof. We first prove that a point $x \in X$ is an isolated point of the level $f^{-1}\{f(x)\}$ if and only if $f$ has a strict maximum or minimum at $x$. The sufficiency of the condition is obvious, and to prove its necessity suppose $f$ does not have a strict maximum or minimum at $x$. In case $f$ has an ordinary maximum or minimum at $x$, it is obvious that $x$ is a limit point of the level $f^{-1}\{f(x)\}$. So let us assume that $f$ does not have even an ordinary maximum or minimum at $x$.

Given an arbitrary neighborhood $U$ of $x$, there exists a cyclicly connected neighborhood $V$ of $x$ such that $V \subset U$. Since $f$ does not have a maximum or minimum at $x$, we can find two points $y$ and $z$ in $V$ such that $f(y)<f(x)<f(z)$. Now $V$ contains a simple closed curve $C$ such that $y, z \in C$, and further $C=A \cup B$, where $A$ and $B$ are two simple arcs from $y$ to $z$ with no other common point. As the sets $A$ and $B$ are connected, so are their images $f(A)$ and $f(B)$, and since each of them contains $f(y)$ and $f(z)$, it follows that $f(x) \in f(A) \cap f(B)$. Thus the level $f^{-1}\{f(x)\}$ contains at least two points of $U$, one of which must be distinct from $x$. Hence $x$ is a limit point of $f^{-1}\{f(x)\}$, which proves the above assertion.

Now, as the space $X$ is second countable, the set $E$ of points of strict maxima and minima of $f$ is countable. Thus the part (b) follows directly from the above assertion. Further, as the levels of $f$ are mutually disjoint, and $X$ is separable, the set $P$ of values of $\alpha$ in $R$ for which $f^{-1}(\alpha)$ has a nonempty interior is countable. The set $Q=P \cup f(E)$ is again countable, and for every $\alpha \in R-Q$ the level $f^{-1}(\alpha)$ is a dense-in-itself boundary set. This completes the proof of the proposition.

As another consequence of the above assertion we have the following

5.14. Corollary. If $X$ is locally cyclicly connected and a connected function $f: X \rightarrow R$ has no strict maximum or minimum, then every level of $f$ is dense-in-itself. 
Added in proof. Regarding Problem 3.11 Dr. Z. Grande has shown in a communication the author that for a real-valued, relatively proper, connected function $f$ on a connected, locally connected, hereditarily normal space $X$, it can be deduced from Theorems 3.3 and 3.6

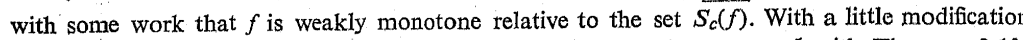
In his argument the following result is obtained which may be compared with Theorem 3.10: if $f$ is a real-valued, relatively proper connected function on a connected, locally connected Hausdorff space $X$, then its restriction to the set $\overline{S_{c}(f)}$ is continuous, Morrey monotone and proper.

The following simplified version of an example communicated by Grande shows that Problem 5.10 does not have an affirmative answer when $X$ is not complete. Let $A$ be the set of rational numbers and $B=R-A$. Then $X=(A \times R) \cup(R \times B)$ is locally connected relative to the induced metric of $R^{2}$. The projection $f((x, y))=x,(x, y) \in X$, is continuous and nowhere monotone, but no point $(x, y)$ in the residual subset $R \times B$ of $X$ is a limit point of the level $f^{-1}\{f((x, y))\}$ along the arc $R \times\{y\}$ that is contained in $X$

\section{References}

[1] G. Choquet, Lectures on Analysis, Vol. I, New York 1969

[2] K. M. Garg. On nowhere monotone functions, I. Derivates at a residual set, Ann. Univ. Sci. Budapest. Eötvös Sect. Math. 5 (1962), pp. 173-177.

[3] - On level sets of a continuous nowhere monotone function, Fund. Math. 52 (1963), pp. 59-68.

[4] - Monotonicity, continuity and levels of Darboux functions, Colloq. Math. 28 (1973), pp. $91-103$.

[5] - On bilateral derivates and the derivative, Trans. Amer. Math. Soc. 210 (1975), pp. 295-329.

[6] R. Hrycay, Noncontinuous multifunctions, Pacific J. Math. 35 (1970), pp. 141-154.

[7] - Weakly Connected Functions, Ph. D. Thesis, Univ. of Alberta 1971.

[8] K. Kuratowski; Topology, Vol. I, New York-London-Warszawa 1966.

[9] - Topology, Vol. II, New York-London-Warszawa 1968.

[10] J. S. Lipiński, Une remarque sur la continuité et la connexité, Colloq. Math. 19 (1968), pp. 251-253.

[11] - On level sets of Darboux functions; Fund. Math. 86 (1974), pp. 193-199.

[12] C. B: Morrey, Jr., The topology of (Path) surfaces, Amer. J. Math. 57 (1935), pp. 17-50.

[13] W. J. Pervin and N. Levine, Connected mappings of Hausdorff spaces, Proc. Amer. Math. Soc. 9 (1958), pp. $488-496$

[14] D. E. Sanderson, Relations among some basic properties of non-continuous functions, Duke Math. J. 35 (1968), pp. 407-414.

[15] W. Sierpiński, Sur un problème concernant les ensembles mesurables superficiellement, Fund. Math. 1 (1920), pp. 112-115.

[16] - Sur l'ensemble des valeurs qu'une fonction continue prend une infinité non dénombrable de fois, Fund. Math. 8 (1926), pp. 370-373.

[17] J. R. Walker, Monotone Mappings and Decompositions, Ph. D. Thesis, Syracuse Univ. 1970.

[18] G. T. Whyburn, Analytic Topology, Amer. Math. Soc. Colloq. Publ., Vol. 28, Amer. Math. Soc., New York 1942

[19] - Dynamic topology, Amer. Math. Monthly 77 (1970), pp. 556-570.

DEPARTMENT OF MATHEMATICS

UNIVERSITY OF ALBERTA

Edmonton, Alberta

Canada

Accepté par la Rédaction le 18. 8. 1975

\section{A perfectly normal locally metrizable non-paracompact space}

by

R. Pol (Warszawa)

Abstract. We construct an example of a perfectly normal locally second - countable and non-paracompact space by a modification of a metrizable space.

The aim of this paper is to describe a construction which by a modification of a metric space yields a locally metrizable, perfect, collectionwise normal and non-paracompact space containing a locally countable non $F_{\sigma}$-set. An application in the dimension theory, given in [4], has been the motivation for such a construction

1. Terminology and notation. We shall use the terminology of [1]. For an ordinal $\alpha$ we shall denote by $D(\alpha)$ the set of all ordinals less than $\alpha$ with the discrete topology and by $W(\alpha)$ the same set with the order topology. The symbol Lim stands for limit countable ordinals. A set $\Sigma \subset W\left(\omega_{1}\right)$ is called stationary if it intersects each closed, cofinal set in $W\left(\omega_{1}\right)$; equivalently (cf. [3], Appendix 1.5), if for each function $\varphi: \Sigma \rightarrow W\left(\omega_{1}\right)$ with $\varphi(\alpha)<\alpha$ there exists $\xi<\omega_{1}$ such that $\left|\varphi^{-1}(\xi)\right|=\kappa_{1}$ If $M$ is a set and $\varrho$ a metric on $M$, then $w(M, \varrho)$ denotes the weight of the metric space $(M, \varrho)$ and $\bar{A}^{\varrho}=\{x \in M: \varrho(x, A)=0\}$ denotes the closure of $A \subset M$ with respect to $Q$. The set of natural numbers is denoted by $N, I$ denotes the unit real interval and $|M|$ stands for the cardinality of a set $M$.

2. The definition of $X$. Let $X$ be a set and $\varrho$ a metric on $X$ such that $w(X, \varrho)$ $=\kappa_{1}$. Suppose that for $\xi<\omega_{1}$ we have given sets $X_{\xi}$ satisfying the following conditions (cf. [5], (3), (4)):

$$
\begin{gathered}
X_{1} \subset \ldots \subset X_{\xi} \subset \ldots \subset X, \quad X_{\xi}^{0}=X_{\xi}, \quad w\left(X_{\xi}, \varrho\right) \leqslant \kappa_{0}, \\
X=\bigcup_{\xi<\omega_{1}} X_{\xi} . \text { and, for } \xi \in \operatorname{Lim}, \quad X_{\xi}=\bigcup_{\alpha<\xi} X_{\alpha}^{0} .
\end{gathered}
$$

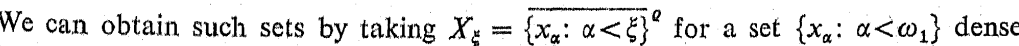
in the space $(X, \varrho)$.

Let us introduce a topology in the set $X$ taking as a base the sets $U \cap X_{\xi}$ where $U$ is open with respect to $\varrho$ and $\xi<\omega_{1}$. By open and closed sets in $X$ we shall understand sets which are open or closed with respect to that topology. 